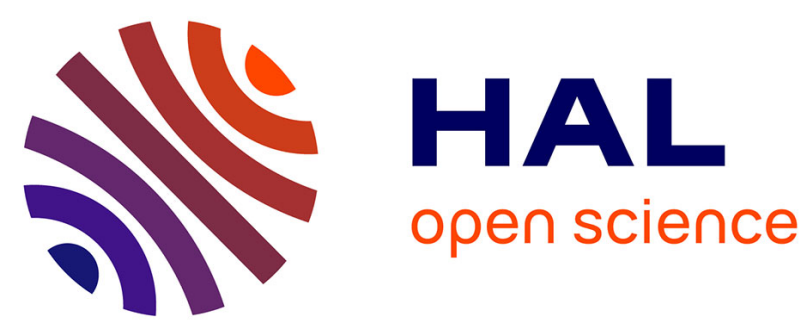

\title{
Multimodal imaging technology by integrated scanning electron, force, and microwave microscopy and its application to study microscaled capacitors
}

Olaf C Haenssler, Sergej Fatikow, Didier Theron

\section{- To cite this version:}

Olaf C Haenssler, Sergej Fatikow, Didier Theron. Multimodal imaging technology by integrated scanning electron, force, and microwave microscopy and its application to study microscaled capacitors. Journal of Vacuum Science \& Technology B, Nanotechnology and Microelectronics, 2018, 36 (2), pp.022901. 10.1116/1.5006161 . hal-03022536

\section{HAL Id: hal-03022536 https://hal.science/hal-03022536}

Submitted on 24 Nov 2020

HAL is a multi-disciplinary open access archive for the deposit and dissemination of scientific research documents, whether they are published or not. The documents may come from teaching and research institutions in France or abroad, or from public or private research centers.
L'archive ouverte pluridisciplinaire HAL, est destinée au dépôt et à la diffusion de documents scientifiques de niveau recherche, publiés ou non, émanant des établissements d'enseignement et de recherche français ou étrangers, des laboratoires publics ou privés. 
Multimodal imaging technology by integrated scanning electron, force, and microwave microscopy and its application to study microscaled capacitors

Olaf C. Haenssler, Sergej Fatikow, and Didier Theron

Citation: Journal of Vacuum Science \& Technology B, Nanotechnology and Microelectronics: Materials, Processing, Measurement, and Phenomena 36, 022901 (2018);

View online: https://doi.org/10.1116/1.5006161

View Table of Contents: http://avs.scitation.org/toc/jvb/36/2

Published by the American Vacuum Society

\section{Articles you may be interested in}

Review Article: Active scanning probes: A versatile toolkit for fast imaging and emerging nanofabrication Journal of Vacuum Science \& Technology B, Nanotechnology and Microelectronics: Materials, Processing, Measurement, and Phenomena 35, 06G101 (2017); 10.1116/1.4992073

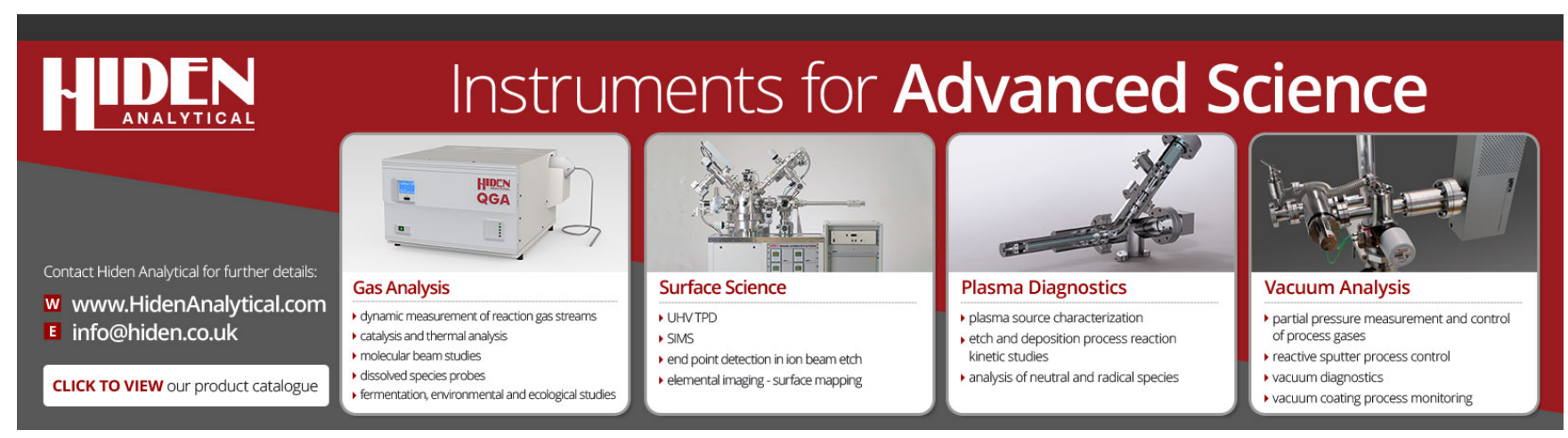




\title{
Multimodal imaging technology by integrated scanning electron, force, and microwave microscopy and its application to study microscaled capacitors
}

\author{
Olaf C. Haenssler ${ }^{a)}$ \\ Department for Computing Science, University of Oldenburg, Oldenburg D-26129, Germany and Institut \\ d'Electronique, de Microelectronique et de Nanotechnologie, CNRS UMR 8520/University of Lille 1, \\ Villeneuve d'Ascq F-59652, France \\ Sergej Fatikow \\ Department for Computing Science, University of Oldenburg, Oldenburg D-26129, Germany \\ Didier Theron \\ Institut d'Electronique, de Microelectronique et de Nanotechnologie, CNRS UMR 8520, Villeneuve d'Ascq \\ F-59652, France
}

(Received 22 September 2017; accepted 11 January 2018; published 25 January 2018)

\begin{abstract}
Extracting simultaneously multimodal nanoscale specimen information, by an integrated microscopy technology, is in the focus of this report. The combination of multiple imaging techniques allows for obtaining complementary and often unique datasets of samples under test. An instrumental setup operating under high-vacuum conditions inside the chamber of a scanning electron microscope (SEM), as a platform fusing various microscopy methods, techniques and processes, illustrates the potential of such multimodal technology. An atomic force microscope based on a compact optical interferometer performs imaging of surface topographies and a scanning microwave microscope records electromagnetic properties in the microwave frequency domain at the same time and spot. An open-source software framework, tailored for vision-based automation by nanorobotics, controls the instrument. The setup allows for simultaneously observing the region-of-interest with SEM resolution, while imaging and characterizing with evanescent microwaves and atomic forces. To validate the approach an analysis of microscale capacitors is included.

Published by the AVS. https://doi.org/10.1116/1.5006161
\end{abstract}

\section{INTRODUCTION}

Performing accurate analysis of surface topography by using both scanning probe microscopy (SPM) and scanning electron microscopy (SEM) is common practice. Combining all these modalities into one hybrid technology to measure a sample and to proof probe integrity in situ without changing the working environment has many advantages. Localization, navigation, live observation of the landing, and scanning process of the probe with the large field of view of an SEM allows for accurate and faster analysis. The use of visionbased nanorobotics control opens the door for automation on the nanoscale through the integration of multidimensional object characterization methods, manipulation strategies applied to probes and samples and image processing techniques, allowing for feature recognition and learning.

Different approaches to integrate atomic force microscopes (AFMs) into scanning electron microscopes are known for over 20 years. In the first report, the electron beam of the SEM was focused onto the edge of the sensing cantilever and a change of number of scattered electrons, caused by the force-induced deflection, were detected. ${ }^{1}$ This modality disables the SEM operation, because the analysis of the cantilever deflection blocks the secondary electron detector for imaging. To investigate nanoelectronic transport processes and cathode-luminescence a combined AFM/SEM based on the laser deflection principle was introduced. ${ }^{2-4}$ A vacuum compatible commercial "Thermo-Microscopes Explorer"

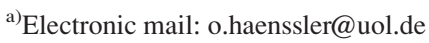

AFM inside an SEM has been used to pick and fix a carbon nanotube between electrodes for further characterization. ${ }^{5}$ Commercial products of laser beam-deflection AFM for the integration into an SEM are available. ${ }^{6,7}$ Another approach is by sensing the atomic forces with tuning forks ${ }^{8}$ or piezoresistive cantilevers. ${ }^{9-12}$ Besides these cantilever readout techniques, optical interferometry was introduced as an AFM technique under ambient environment since the beginning of the invention ${ }^{13}$ and also as an ultrahigh vacuum version. ${ }^{14}$ The force sensitivity is down to the attonewton range by using soft cantilever. ${ }^{15}$ The optical path and hence the parts of a Michelson-Interferometer requires less space than the beam deflector solution. It is therefore favorable if only little room is available, as inside the vacuum chamber of an SEM, and furthermore to establish in this work nanoautomation techniques. The ease of adjustment and the opportunity to eliminate electro-magnetic disturbances by locating the electronics outside the region of measurement are other advantages, especially when probing with high frequency electromagnetic fields. All these methods enable conventional AFM-based surface analysis inside an SEM, which means one cantilever, one application and no possibility to use microwave evanescent waves as a sensing modality in a vacuum.

By the combination of a scanning tunnel microscope (STM) with a microwave resonator, ${ }^{16}$ this "radio frequency SPM" technique has been evolved to commercially available scanning microwave microscope (SMM) after Ash and Nicholls developed the first scanning near-field microwave microscope. ${ }^{17}$ Such microscopy mode at air is more and more settling in the field of characterizing complex electric 


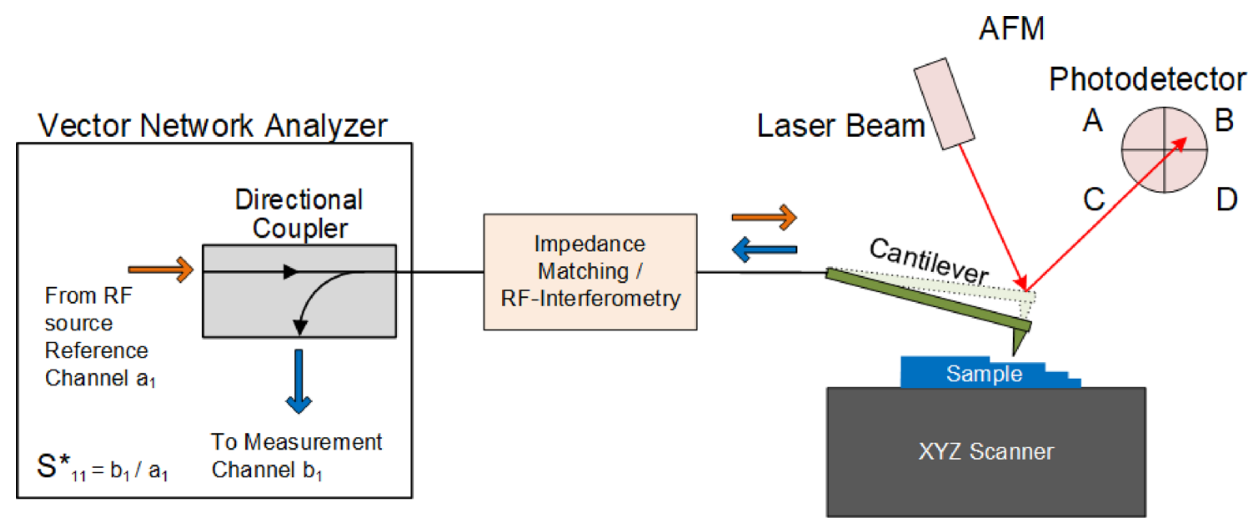

FIG. 1. (Color online) Basic parts of a scanning microwave microscope, here based on a laser deflection AFM (Laser and 4-Quadrant Photodetector) probing a sample via a conductive cantilever.

properties of different nanomaterials. ${ }^{18,19}$ This exclusive type of SPM is able to image and quantify local dielectric and conductivity measurements in the range of 1 to $60 \mathrm{GHz} .{ }^{20} \mathrm{SMM}$ bridges the spectral frequency gap between SPM measuring with DC or low frequency currents on one side and terahertz $\mathrm{STM},{ }^{21}$ respectively, near-field scanning optical microscopes (NSOM) ${ }^{19,22}$ on the other side. An SMM consists of two instrumental parts: An AFM or STM and a microwave source with a gain-phase detection unit, often a vector network analyzer (VNA). The conductive probe is electrically connected to one port of such a VNA. In case of an AFM, the cantilever deflection, caused by atomicscaled force interaction at the tip-sample junction, or in case of an STM, the tunneling current, records synchronously to the electromagnetic information obtained by a VNA. This information is the response of an incident evanescent reference wave $a_{1}$ introduced to the sample by the probe, reflected back and VNA-internally split by a high frequency directional coupler as wave $b_{1}$ (Fig. 1). For the types of nanoelectronic samples or objects studied with an SMM, the complex scattering parameters contains information about the complex quantities of conductivity, permittivity, and permeability. Extracting this information out of the magnitude and phase information of $S_{11}$ and $S_{21}$ at the nanoscale is one challenge. An understanding of both the interaction of the incident electromagnetic wave and the physics of the sample is needed to obtain the quantitative data from the measurements. The impedances here are in the order of quantum wire resistance of around $25.8 \mathrm{k} \Omega$. In order to overcome the "50 $\Omega$ hurdle", some efforts to improve the measurement setup are required in order to achieve high sensitivity in the high frequency range with high resistances in the kilo-ohm range. ${ }^{23}$ Matching the impedance of the measurement system to the sample-under-test (SUT) at a single frequency is one possibility. RF-Interferometry is another approach that enhances the system sensitivity significantly (Fig. 1, circuit block between VNA and cantilever). ${ }^{24}$ In each interval of the constantly repeating interference frequency, it is possible to take data samples over a broad frequency range.

The main research targets of SMM are the characterization of hetero-structured devices mostly in semiconductor research and growingly in cell biology. An overview of reported applications, divided into imaging and quantitative measurements alongside topography imaging, follows (Table I).

TABLE I. Reported targeting applications of scanning microwave microscopy.

Thin film imaging

1-layer to 3-layer graphene (Refs. 25 and 26), 1-layer hexagonal boron nitride ("white graphene") (Ref. 27)

Chromium on glass substrate (Refs. 28 and 29)

Boron-doped silicon (Refs. 30 and 31)

Vanadium dioxide $\left(\mathrm{VO}_{2}\right)$ and chemical-vapor-deposition graphene (Ref. 32)

Phosphorus ions implanted into p-type silicon substrate (Ref. 33)

Multilayer ferrite on glass substrate (Ref. 34)

Transition metal dichalcogenide monolayers (Ref. 35)

Organic self-assembled monolayers of alkane-thiols (Ref. 36)

Yttrium iron garnet magnetic films (Ref. 37)

Nanowire imaging

$\mathrm{In}_{2} \mathrm{Se}_{3}$ nanoribbons (Ref. 38)

GaN nanowires (Ref. 39)

Biological cell imaging

Human monocytic leukemia cells (THP1) on gold and glass substrates (Ref. 40)

Epidermal cells of Yucca filamentosa (Ref. 41)

C2C12-Myoblast cells (muscle cells) interacting with multiwall carbon nanotubes (Ref. 42)

Escherichia coli cells (Ref. 43)

Yeast cells behind a membrane (Ref. 44)

3D-material imaging

Buried metal structures (Ref. 45), metal-insulator-semiconductor systems (Refs. 38 and 46), and single phosphorous layer (Ref. 47)

Coal (Ref. 48)

Quantitative measurements

Nanoscaled capacitances (Refs. 24 and 49) and interconnects (Ref. 50) in the sub-fF range

Dopant concentrations in n-type Si (Ref. 51), p-type Si (Ref. 52), and Si p-n junctions (Ref. 53)

Permittivity of dielectric materials (Refs. 54 and 55) and polar liquids (Ref. 56)

Conductivity of superconductors $\mathrm{YBa}_{2} \mathrm{Cu}_{3} \mathrm{O}_{7}$-d (Ref. 57), niobium-based thin films (Ref. 58), and hematite and calcite minerals (Ref. 59) 
This compilation presents works conducted by SMM in standard nonvacuum laboratory environment. The mentioned work on superconductors ${ }^{57}$ used an STM-assisted SMM in a cryogenic environment. Integrating an AFM-based SMM into an SEM leads to several benefits. Reducing the water meniscus at the AFM-Tip through vacuum environment to enhance the spatial resolution, ${ }^{60}$ monitoring the probe tip quality with an SEM without removing it out of the vacuum workspace, ${ }^{61}$ obtaining additional dimensional information to reduce uncertainty at characterization of different aspects $^{62}$ and in situ manipulation of the samples ${ }^{5}$ in an open-source nanorobotics environment. This article describes such a multimodal scanning microscopy technology in Sec. II, validates it setup by investigating microscaled capacitors in Sec. III and concludes with an outlook.

\section{EXPERIMENTAL METHODS}

The vacuum-compatible instrumental system, the scanning electron, force and microwave microscope $\left(\mathrm{SEM}^{2}\right)$, based on the following parts: (1) SEM (commercial), (2) scanning/AFM (self-designed/-built), (3) SMM (selfdesigned/-built), (4) vector network analyzer (commercial), (5) sample stages, fine and coarse positioning (selfdesigned), (6) controller (self-built) for AFM and SMM, and (7) control software with graphical user interface (selfdesigned/-built, based on open-source nanorobotic software framework). Figure 2 shows on the left the block diagram of this hybrid microscope with the functionality of probing samples with low power electro-magnetic high-frequency evanescent waves, electron beams, and atomic forces. Due to the construction of the probe tip, electric evanescent waves with tip geometry-dependent exponential decay lengths are propagating in the less dense medium. The setup works in a Zeiss LEO1450 tungsten SEM, equipped with a secondary electron detector and three cameras. The scanning parts are mounted on a dovetail stage-adapter for fast setup exchange. An USB3.0 sideview infrared camera records the tip-sample approach. To measure the cantilever deflection an optical interferometric approach was deployed. This information is fed to an AFM/SMM-controller which closes the loop by steering the piezodriven AFM-Scanner (PicoCube/Physik Instrumente), while this is coarse positioned by a piezodriven stage (SLC Series/SmarAct). A Rhode and Schwarz VNA ZVA24 is connected to the conductive tip and generates and detects the microwave signals. The software control and scan-image acquisition of the instrument is adopted as an open source software framework tailored for nanorobotics and -automation. ${ }^{63}$

\section{A. Laser interferometer AFM-in-SEM}

To obtain information about the surface topography a vacuum compatible AFM based on an optical Michelson interferometer (PicoScale/SmarAct) was designed (Fig. 3) and incorporated into the SEM. The infrared laser is working at a wavelength of $1532 \mathrm{~nm}$ (NIST-traceable) with a modulation frequency of $30 \mathrm{MHz}$. The light energy is coupled via optical fiber into an integrated optics head of $6.4 \mathrm{~mm}$ (a)

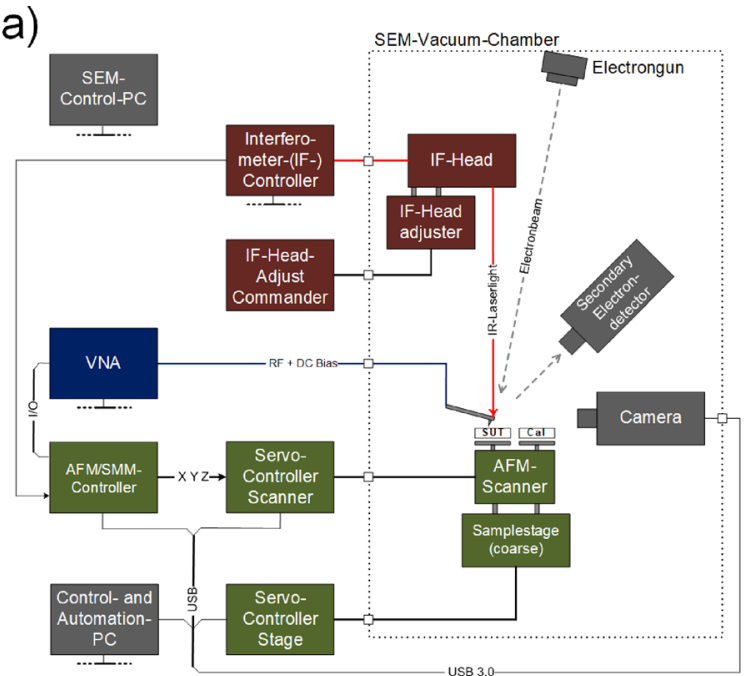

(b)

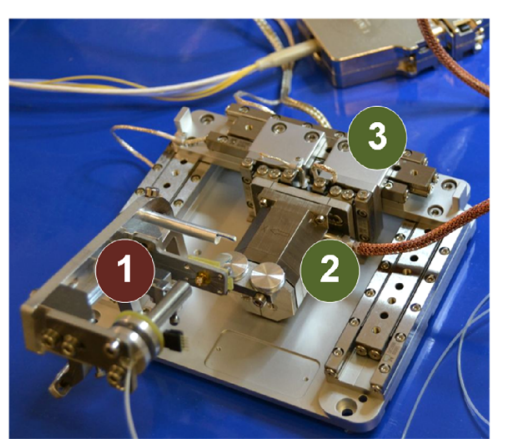

FIG. 2. (Color online) (a) Schematic of the Scanning Electron, Force and Microwave Microscope $\left(\mathrm{SEM}^{2}\right)$ with (i) optoelectronic parts of the Laser interferometer (IF-) AFM, the AFM/SMM-scanner-controller, and the samplestage, (ii) microwave signal source and detection by a VNA, and (iii) image-live-acquisition by SEM, optical camera and PC's. The dotted line illustrates the vacuum chamber of the SEM. (b) Photograph of probing head (1), AFM-scanner (2), and sample stage (3).

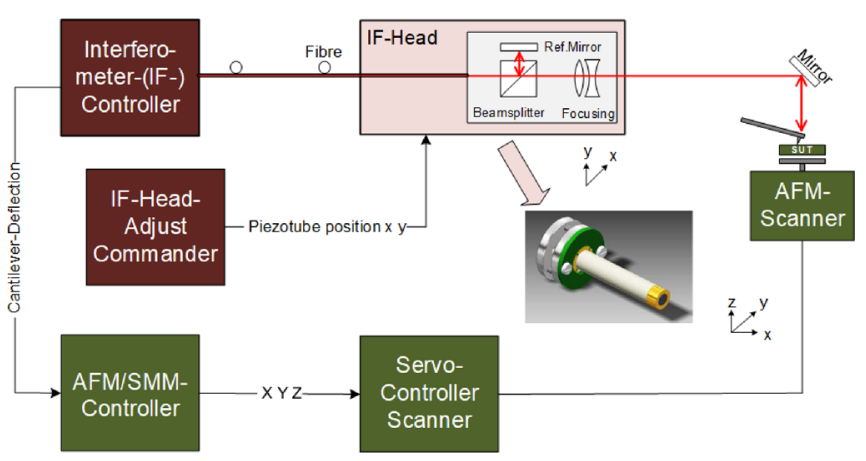

FIG. 3. (Color online) Schematic of the control loop of the laser interferometer AFM-in-SEM. An optical fiber guides the modulated laser light to the interferometer-head. The IF-head houses the optics, splitting the beam into a reference and a sensing beam. This beam is than collimated and focused onto the AFM-cantilever. The IF-controller generates a signal proportional to the cantilever deflection. This feeds the AFM/SMM-controller to move the AFM-scanner stage accordingly. A piezotube actuator, steered by the IFhead-adjust-commander, fine positions the laser beam inside the vacuum chamber with two degree-of-freedom. Inset (middle): CAD image of the steerable IF-head. 


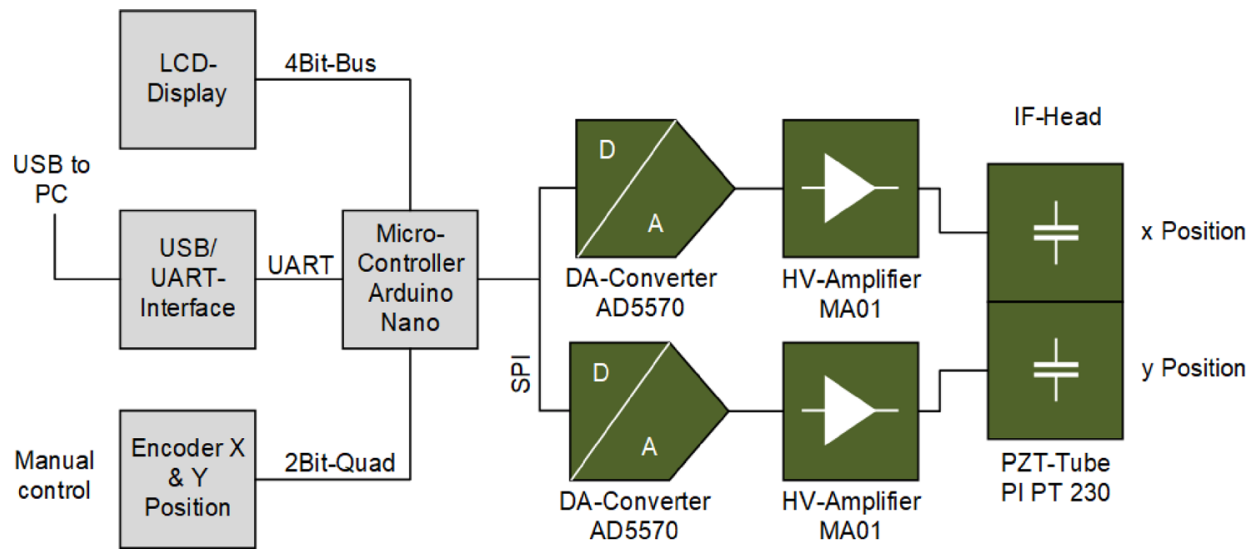

FIG. 4. (Color online) Block diagram of the IF-head-adjust control commander. Two analog voltages for $\mathrm{x}$ and y position movements are generated by a microcontroller and amplified to high voltages. The four segmented PZT-tube houses and moves the IF-head optics, respectively, the laser beam to align it to the reflective surface of the cantilever.

diameter (IF-Head in Fig. 3). The focused laser light shines onto the reflective side of the platinum cantilever. The spot size is $50 \mu \mathrm{m}$. While scanning the measured deflection, the information of the cantilever feeds the AFM/SMM-controller to move the AFM-Scanner following the topography of the SUT. The AFM act as a sample-scanner to reduce cable induced vibrational-modulation effects to the microwave signals. The IF-Head allow for steering the laser beam electronically by piezo ceramics inside the SEM chamber (inset in Fig. 3). The piezo actuator tube (PT230.14/Physik Instrumente) has four segmented electrodes with maximum $14 \mu \mathrm{m}$ end tip travel. To move the lead zirconate titanate (PZT) tube in $\mathrm{x}$ and $\mathrm{y}$, two high-voltages are applied. These voltages are generated by a self-built IF-Head-Adjust Commander (see Fig. 4) with low voltage noise and manual/ remote-control (MATLAB/PYTHON) capabilities. A microcontroller (ATMega 328/Atmel) controls two 16 Bit-Digital-toAnalog Converters (AD5570/Analog Devices, Corp.) as their output voltages are converted up to maximum $\pm 150 \mathrm{~V}$ by high voltage amplifiers (MA01/Apex). The drift and noise of the generated high-voltages results to an additional error in movement of $3 \mathrm{~nm}$ at the tube end after $4 \mathrm{~h}$ operation, confirmed by interferometric measurements (Double-Beam Plane-Mirror Interferometer SP2000D/SIOS). The thermomechanical noise detection of one cantilever resonance frequency in vacuum, resulted in $16.074 \mathrm{kHz}$ with an amplitude of $195 \mathrm{pm}$ (RMS) and due to higher damping at air of $27 \mathrm{pm}$.

\section{B. Near-field microwave probe}

The conductive probing tip (25Pt300/Rocky Mountain) acts as an electrically small antenna, radiating nonpropagating evanescent fields. On interaction with the sample, the field distribution changes, under the assumption that the tip-sample distance and the tip radii are much smaller than the wavelength $\lambda$ of the incident microwave. The interface between the cantilever substrate and the wiring to the VNA is a critical wave path, also in terms of transmission losses. A waveguide structure was designed on an RF substrate (RO4003C/Rogers) matching the impedance of the cantilever substrate, acting as a transmission line, to the connector (see

Fig. 5). The impedance of the 25Pt300 substrate with the platinum cantilever signal line and a ground layer under the bottom of the ceramic substrate is calculated to $74 \Omega$ in microstrip mode according to the given manufacturer's specifications. Modal driven electromagnetic simulations (HFSS/ Ansoft) optimized the transmission and return losses in the frequency range 1 to $10 \mathrm{GHz}$ of the near-field microwave probe from the entrance of the RF-connector via a bonding wire to the probe substrate. The simulation of the near-field probe was carried out under two constructive design constraints: First of all, an RF connector (SMP/Rosenberger) has been selected with the lowest possible engagement force and

(a)

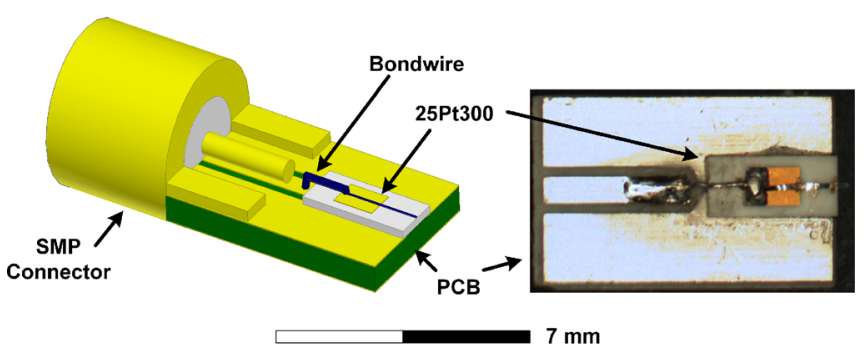

(c)

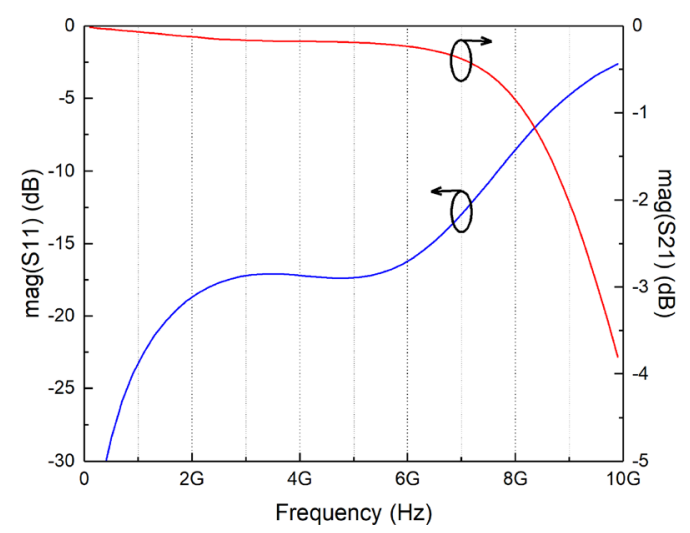

FIG. 5. (Color online) Near-field microwave probe: (a) isometric computeraided design view, (b) photograph, and (c) simulation results of the magnitudes of scattering parameters: transmission loss $\left[\operatorname{mag}\left(\mathrm{S}_{21}\right)\right]$ and return loss $\left[\operatorname{mag}\left(\mathrm{S}_{11}\right)\right]$ over frequency. 
return losses of better $26 \mathrm{~dB}$ up to $24 \mathrm{GHz}$, the maximum operating frequency of the VNA. Second, a RMN 25Pt300 cantilever is used due to comparability of measurements with a commercial SMM. In order to join the connector and the cantilever together with low losses, a transmission line circuit board has been designed under nonoperative conditions. For this purpose, the free standing part of the cantilever, with a length of $300 \mu \mathrm{m}$, was cut off for the simulation of $S_{11}$ and $\mathrm{S}_{21}$ and a waveport was placed at this point, as well as at the connector entrance. A shielded air box surrounded the transmission line in $4 \mathrm{~mm}$ distance.

The maximum operating frequency with the commercial 25Pt300 probe turned out to be $7 \mathrm{GHz}$. Here, the transmission $\mathrm{S}_{21}$ and return losses $\mathrm{S}_{11}$ becomes to maximum -0.5 and $-12.5 \mathrm{~dB}$, respectively, in a $50 \Omega$ back-to-back simulation.

\section{Instrument control}

Acquire the scan data topography and complex SParameter synchronously in time and space is the basis of the control of the experimental setup. An additional requirement is to embed the $\mathrm{SEM}^{2}$ system into a robotic environment for future automated characterization, handling, and manipulation tasks, as tip sharpening by focused ion beam, image-guided measurements, and automated calibration kit positioning. The home-built AFM/SMM-controller incorporates an field programmable gate array (Zynq-7020/Xilinx) for such software control requirements and is capable of controlling both microscopy modalities, atomic force and nearfield microscopies. ${ }^{64}$ The acquired data stream into the aforementioned nanoautomation software framework, such as live camera frames. This allows for image-based robotic control of the sample positioning, too. Due to software performance reasons of the framework, the live acquisition of the SEM images here was switched off, while the SEM Control personal computer still controlled and acquired SEM scan images.

\section{Microscaled capacitors}

Arrays of metal-oxide-semiconductor (MOS) capacitors validate the proof-of-concept of the imaging technology.
Due to the geometric aspect ratios in such scales, the fringing fields, originating from the sidewall edges and the top of the upper plate of microscaled capacitors, has to be taken into consideration. ${ }^{65} \mathrm{~A}$ sketch of the photolithographically fabricated capacitors with the field lines for illustration is shown in Fig. 6. The patterning on top of the $\mathrm{SiO}_{2}$ dielectric was done by $\mathrm{Au}$ e-gun evaporation. To form a contact for grounding, aluminum was evaporated on the back.

Several approaches to determine the value of microscaled MOS capacitors has led to numerous approximation formulae. ${ }^{66-73}$ The reference used in all studies to calculate the total capacitance, used a complex approximation conformal mapping technique based on the Schwarz-Christoffel transformation. ${ }^{74}$ A simplified formula of capacitance over unit length c was introduced by Chuang et al. [Eq. (1)]. ${ }^{73}$ It took into account the sidewall and top-surface fringing field effects for rectangular microbeam structures

$$
\mathrm{c}=\varepsilon *\left[\left(\frac{\mathrm{w}}{\mathrm{h}}\right)-1.06+3.31\left(\frac{\mathrm{t}}{\mathrm{h}}\right)^{0.23}+0.73\left(\frac{\mathrm{w}}{\mathrm{t}}\right)^{0.23}\right],
$$

where $\mathrm{w}$ is the width of the top plate, $\mathrm{h}$ is the height distance between the top and the lower ground plates forming the capacitor, $\mathrm{t}$ is the total thickness of the top plate, and $\varepsilon$ is the permittivity of the dielectric. The geometrical range of validity is given to $\mathrm{w} / \mathrm{h}>0.1$ and $0.1<\mathrm{t} / \mathrm{h}<10$. Using finite element electromagnetic simulation, the theoretical values of the used capacitances were derived. Based on Eq. (1), the following approximation for the round microscaled capacitors in the used geometrical range was found. The first term describes the parallel plate contribution, follows compensation term, sidewall and top plate fringe portions

$$
\begin{aligned}
\mathrm{C}_{\text {Micro Cap }}= & \varepsilon * \mathrm{~W} *\left\{\left(\frac{\mathrm{w}}{\mathrm{h}}\right)+3.3+0.02\left(\frac{\mathrm{t}}{\mathrm{h}}\right)^{0.23}\right. \\
& \left.+0.02\left(\frac{\mathrm{w}}{\mathrm{t}}\right)^{0.23}\right\} * \pi / 4 .
\end{aligned}
$$

The results of a correlation of known approaches, simulation analysis, and the approximation Eq. (2) for the

\section{(b)}

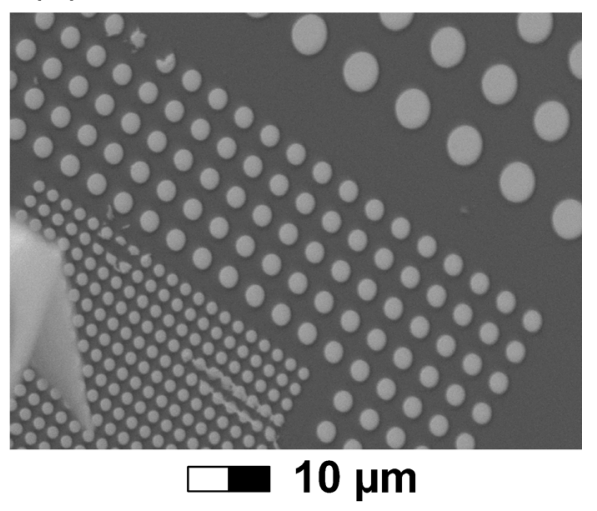

FIG. 6. (Color online) (a) Cross-sectional view of the investigated microcapacitor with electric field lines for illustration of the fringing effect. (b) SEM image showing three types of microcapacitors arrays during scanning with a probe tip (lower left corner). 


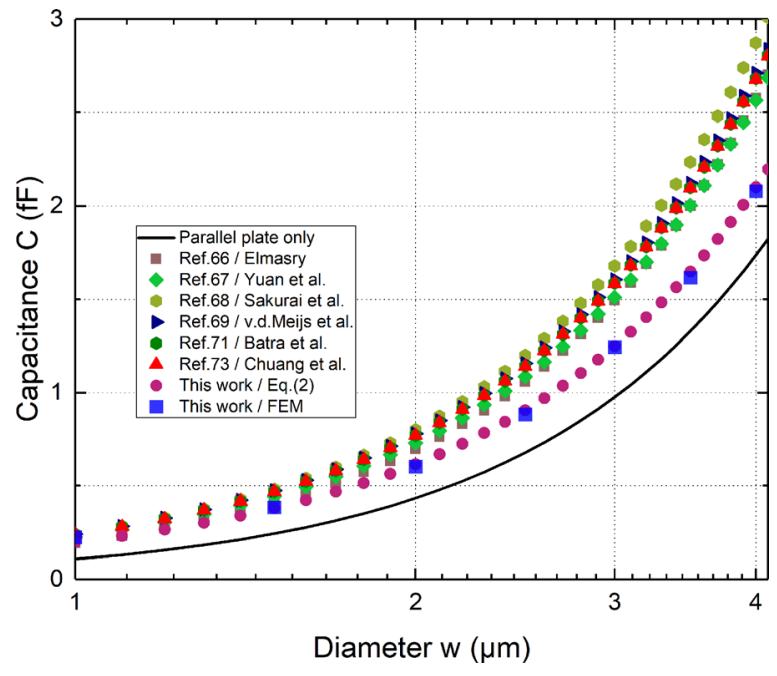

FIG. 7. (Color online) Capacitance values as a function of the round dot diameter with $250 \mathrm{~nm}$ thick $\mathrm{SiO}_{2}$ as dielectric and a $125 \mathrm{~nm}$ thick top plate electrode. Different electrostatic field approximations formulae (Refs. 66-69, 71, and 73) are compared to finite element method analysis and Eq. (2).

structures used in this study are shown in Fig. 7. The values of the rectangular parallel plate capacitor without fringing effect indicate the solid line.

In this work, round capacitor arrays of three diameters ( $\mathrm{w}=1.5,2$, and $4 \mu \mathrm{m}$ ) with the same $\mathrm{SiO}_{2}$ dielectric height $(\mathrm{h}=250 \mathrm{~nm})$, top plate thickness $(\mathrm{t}=125 \mathrm{~nm})$ were used for imaging. Their calculated correspondent values become $380 \mathrm{aF}, 615 \mathrm{aF}$, and $2.10 \mathrm{fF}$. By using the approximation, ${ }^{66}$ differences to the results by Eq. (2) is found to be $5 \%, 7 \%$, and $10 \%$, respectively, for the given capacitor values.

\section{RESULTS}

The $\mathrm{SEM}^{2}$ operated in contact mode under high vacuum conditions $\left(7 \times 10^{-6} \mathrm{mbar}\right)$. Due to the limitation of the AFM-scanner stage of $5 \times 5 \times 5 \mu \mathrm{m}$ in $3 \mathrm{D}$ space, the maximum area of measurement is restricted. The sample-undertest incorporated, in addition to the microscaled capacitors in the center for the SMM study, alignment and finding structures for light and electron microscopy and for image-based robotic navigation. ${ }^{75}$ This multimodal sample is to adjust in electron microscopy mode, the magnification, which has typical errors in the range of 5\%-10\%, and the astigmatism. Scanning was done by 1 line/s at a resolution of $128 \times 128$ pixel and an RF bandwidth of $1 \mathrm{kHz}$. The smallest fabricated capacitors (target diameter $1.5 \mu \mathrm{m}$ ) are showing topography differences in diameter and top flatness. As a reference, the capacitors were studied with a commercial JPK NanoWizard AFM [Fig. 8(b)]. It works under ambient condition and probes with a different cantilever (Tap300/Budget Sensors), having doubled high-spring constant $(40 \mathrm{~N} / \mathrm{m})$ in regards to the near-field microwave probe cantilever. The figure shows the spatial distribution of topography of two different areas of a total array of $21 \times 211.5 \mu \mathrm{m}$ capacitors. Topography line profiles of two capacitors (Fig. 9: both lower right capacitors) yields to physical dimensions between 1.5 and $1.7 \mu \mathrm{m}$ in diameter and $125 \mathrm{~nm}$ in height. Due to differences in height, the results of the $\mathrm{SEM}^{2}$ are corrected by a factor of 1.3 , matching the height of $125 \mathrm{~nm}$ given by the commercial AFM measurement as a reference height. The top plateau swale varies up to $40 \mathrm{~nm}$ (Fig. 9) for different capacitors and cannot be investigated in SEM without destructive testing by ion etching methods. For this purpose, the SEM would have to be equipped with a focused ion beam milling unit, and then it is possible to conduct a cross section cut through the capacitor for inspection purposes by means of ion etching.

In the microwave regime, changes in complex impedance on the top were detected, by measuring the reflection coefficient with the near-field microwave probe and the VNA (Fig. 10). An electric interferometric approach, by using an optional feature in the Rhode and Schwarz VNA of differential signaling ("True Differential Mode"), resulted in sensitivity enhancement at a limited number of single interference frequencies. This implies that these sensitivity enhancing measurements can only be made at or near the repeating interferences. However, these interferences, with a separation of $72.6 \mathrm{MHz}$, span over the entire operating range of the near-field probe. The measurement path to the probe is one part of the internal differential signal and unchanged. The second part of the differential signal, which can be shifted internally in the VNA itself in amplitude and phase (a)

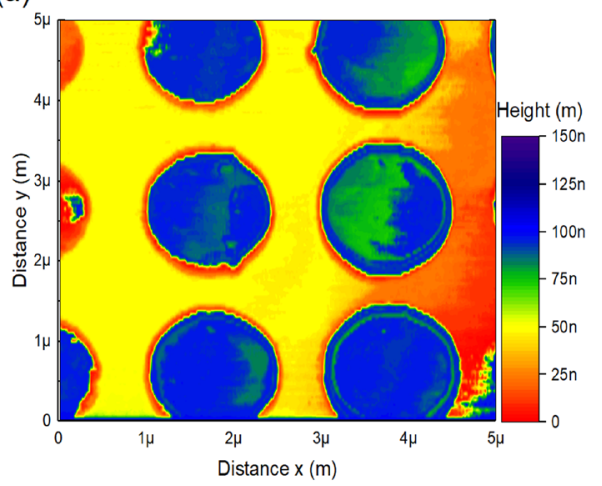

(b)

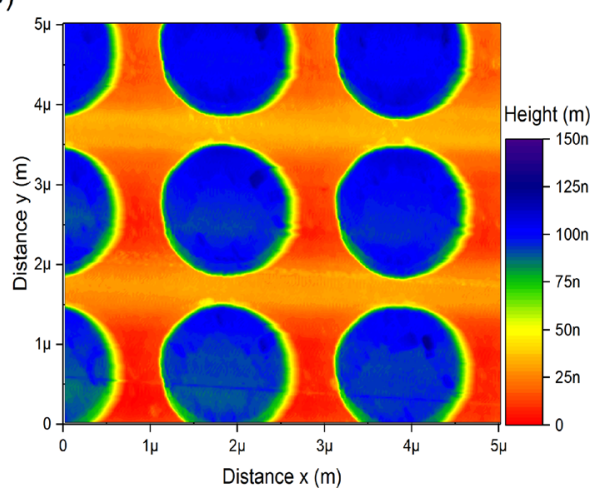

FIG. 8. (Color online) Spatial distribution of topography by (a) SEM ${ }^{2}$ and (b) JPK NanoWizard AFM. Data manipulation by GWYDDION: Mean plane subtraction and line-by-line matching of height median. The right-hand color bar refers to the respective height value. 


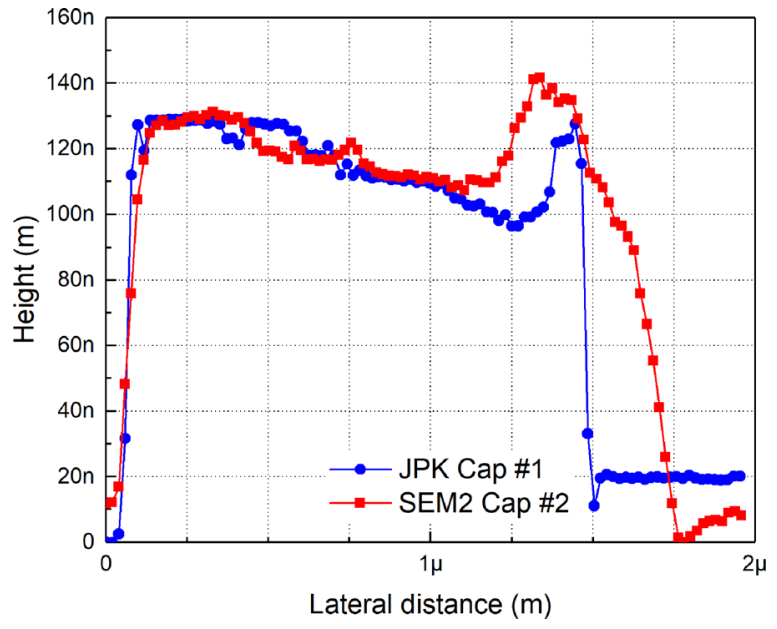

FIG. 9. (Color online) Topography line scan of two different microcapacitors with $\mathrm{SEM}^{2}$ and JPK NanoWizard. Variations in diameter and top plate roughness are observable.

(a)
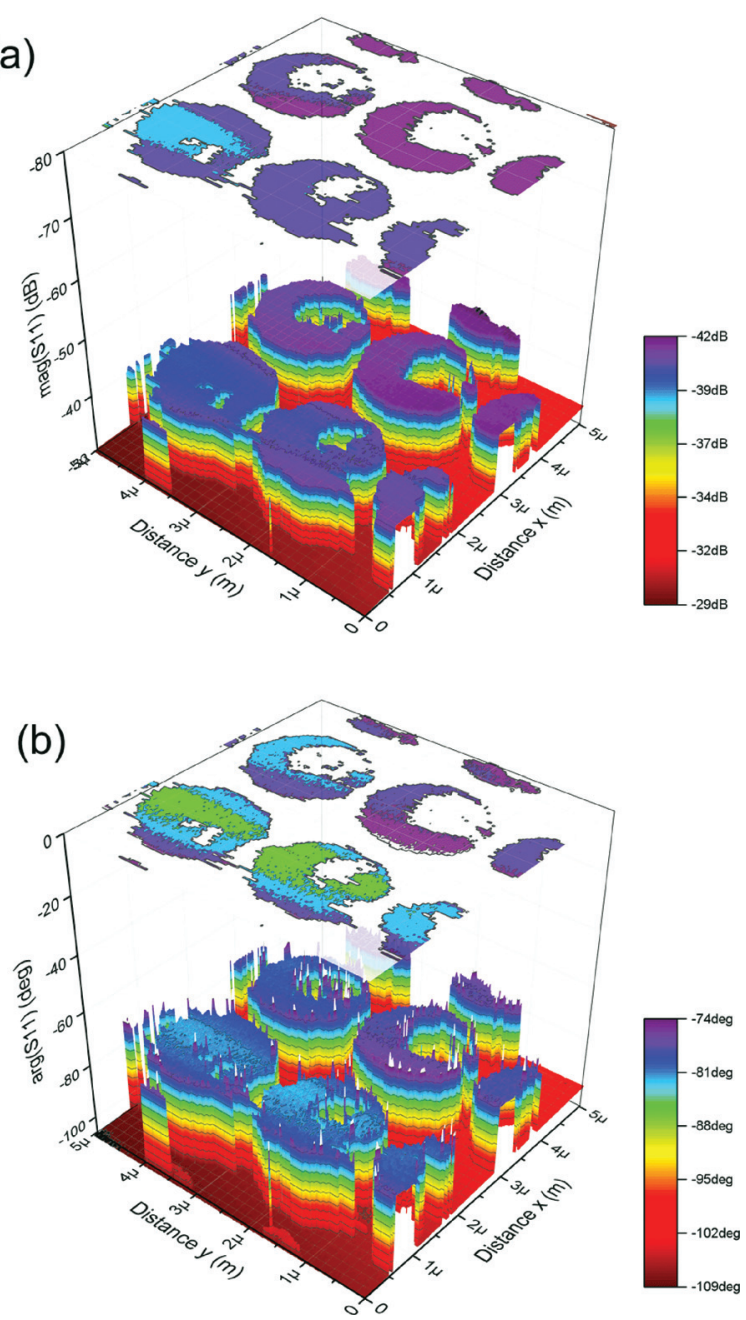

FIG. 10. (Color) 3D color and top projection contour map of (a) magnitude (mag) and (b) phase (arg) of reflection coefficient $\mathrm{S}_{11}$ measured by $\mathrm{SEM}^{2}$ at $4.8 \mathrm{GHz}$ of an area with $1.5 \mu \mathrm{m}$ capacitors. Data manipulation by GWYDDION: Mean plane subtraction. The right-hand color bar refers to the respective measurement value of the scattering parameter $S_{11}$. in regards to the first part, represents the reference path or arm of the interferometer. This signal is internally attenuated by $1.84 \mathrm{~dB}$ and shifted in phase in order to obtain a sharp minimum at $1 \mu \mathrm{m}$ height above the sample surface. This height was selected exclusively to have fixed standard conditions between different measurements. The output power of the VNA has been set to $0 \mathrm{dBm}$ in all measurements. According to simulation, electrical field strengths of 3 $\times 10^{4} \mathrm{~V} / \mathrm{m}$ are calculated at the tip of the probe. Scanning over the $1.5 \mu \mathrm{m}$ capacitors at $4.769 \mathrm{GHz}$ reveals a downshift of the interference frequency of $315 \mathrm{kHz}$. This leads on the other hand, to an increase of $12 \mathrm{~dB}$ magnitude of $S_{11}$ at the fixed measurement frequency during the scan. At $9.996 \mathrm{GHz}$ we found at the same capacitor area a shift of $866 \mathrm{kHz}$, but the magnitude $S_{11}$ increased only up to $2 \mathrm{~dB}$ of the interference minimum of $-42 \mathrm{~dB}$. As mentioned in Sec. II B by simulation, the maximum working frequency of the near-field probe is $7 \mathrm{GHz}$.

In two microscope modalities, AFM topography and electromagnetic microwave properties, it can be seen that the top plate part of the fabricated capacitors features strong imperfections in terms of roughness. Exemplarily measured at one capacitor, the depression on top found to be in magnitude $\left(\mathrm{S}_{11}\right) \approx 0.5 \mathrm{~dB}$ and phase $\arg \left(\mathrm{S}_{11}\right) \approx 4^{\circ}$ at $4.8 \mathrm{GHz}$ (Fig. 11). The electrical roughness can be separated in two parts: (1) the contribution of the topography roughness, which may change the parasitic stray capacitance between the probe and ground, and (2) for the same height, the different contributions of the stray capacitance originating from the top electrode and from the rest of the sample. The electric field is screened by the top plate electrode and while scanning, this screening effect is at maximum, near the capacitor center. This means that the electric field of the stray capacitance between the cantilever and ground is partly shielded from the top plate electrode during scanning. Whereas this does not occur so strongly at the edges, this shielding effect toward the center is maximum. In this case, however, the top electrode is not symmetrical to the $2 \mathrm{D}$ axis.

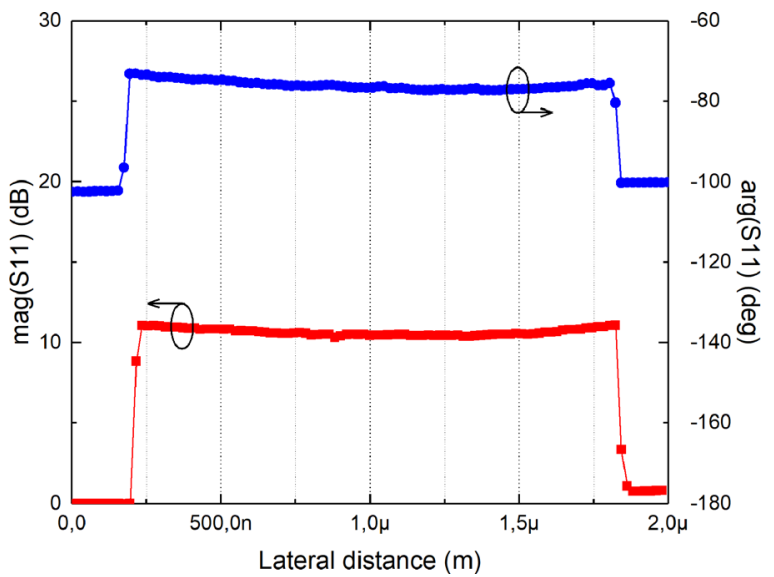

FIG. 11. (Color online) Microwave sectional profile measured by $\mathrm{SEM}^{2}$ at $4.8 \mathrm{GHz}$ of one microcapacitor. Magnitude and phase of reflection coefficient, $\operatorname{mag}\left(\mathrm{S}_{11}\right)$ and $\arg \left(\mathrm{S}_{11}\right)$, respectively. 


\section{SUMMARY AND CONCLUSIONS}

A hybrid technology by integrating several microscopy modalities gaining multiple information of sample surfaces by detection of light, electron, atomic force, and microwave interaction was presented. A study of microscaled capacitors evaluated an instrumental platform, showing the potential of this multimodal technology. The capacitance approximation formulas for rectangular MOS structures known from literature deviated from the RF simulation results by more than $20 \%$, despite the transformation into round areas. For this reason, an approximate formula for round capacitor plates is developed, for the discussed MOS geometries. Performing quantified measurements by using calibration methods with known capacitor values or different heights (see Refs. 36 and 55, respectively,76) was not in the scope of this report. Capacitance values in the calculated area of $380 \mathrm{aF}$, showed strong contrast proofing the concept. Smaller scaled features were not available at the time of preparation. Future work will focus on quantified SMM measurements by utilizing a modeling and de-embedding process. ${ }^{77}$

Vison-based open-source robotics and automation on the nanoscale, enables future additional methods to extend the knowledge of samples and materials, to tool SPM probes and to manipulate nanoscale objects. Arbitrary scanning trajectories, free programmable with our own-designed AFM/ SMM-controller, will result in localized sample-specific dynamic data densities from the region-of-interest. Spectroscopic imaging, voltage or frequency as two examples, with the help of adaptive image processing methods, will be performed to study the properties of novel materials. Joint analysis of multidimensional microscopic data sets is mandatory to enhance the calibration quality to obtain quantified data, not only in near-field microwave probing. Multidimensionality promise to widen the knowledge of materials, but, on the other hand, it leads to Big-Data challenges. These can be solved by employing machine-learning methods.

\section{ACKNOWLEDGMENTS}

This work was supported by the bilateral programme of Agence Nationale de la Recherche (ANR) and the Deutsche Forschungsgemeinschaft (German Research Foundation, DFG) (Project GZ: FA347/48-1) and under the ANR program Equipex (ExCELSiOR). The authors thank IESLFORTH, Heraklion, Greece, for manufacturing the calibration sample.

${ }^{1}$ A. V. Ermakov and E. L. Garfunkel, Rev. Sci. Instrum. 65, 2853 (1994).

${ }^{2}$ M. Troyon, H. N. Lei, Z. Wang, and G. Shang, Microsc. Microanal. Microstruct. 8, 393 (1997).

${ }^{3}$ K. Fukushima, D. Saya, and H. Kawakatsu, Jpn. J. Appl. Phys., Part 1 39, 3747 (2000).

${ }^{4}$ I. Joachimsthaler, R. Heiderhoff, and L. J. Balk, Meas. Sci. Technol. 14, 87 (2003)

${ }^{5}$ P. A. Williams et al., Appl. Phys. Lett. 80, 2574 (2002).

${ }^{6}$ F. Hang, D. Lu, and A. H. Barber, Mater. Res. Soc. Symp. Proc. 1187, 135 (2009).
${ }^{7}$ M. Bolorizadeh, F. Panteleit, and F. Hitzel, Proceedings of the Microscopy and Microanalysis Conference, Indianapolis, IN, 4-8 August (2013), Vol. 19, pp. 1300-1301.

${ }^{8}$ A. Lewis, A. Komissar, A. Ignatov, O. Fedoroyov, and E. Maayan, Microsc. Microanal. 21, 1433 (2015).

${ }^{9}$ U. Stahl, C. W. Yuan, A. L. de Lozanne, and M. Tortonese, Appl. Phys. Lett. 65, 2878 (1994).

${ }^{10}$ U. Mick, V. Eichhorn, T. Wortmann, C. Diederichs, and S. Fatikow, Proceedings of the IEEE International Conference on Robotics and Automation, Anchorage, AK, 3-8 May (2010), pp. 4088-4093.

${ }^{11}$ J. Kreith, T. Strunz, E. J. Fantner, G. E. Fantner, and M. J. Cordill, Rev. Sci. Instrum. 88, 53704 (2017).

${ }^{12}$ T. Angelov et al., J. Vac. Sci. Technol., B 34, 06KB01 (2016).

${ }^{13}$ R. Erlandsson, J. Vac. Sci. Technol., A 6, 266 (1988).

${ }^{14}$ B. Kracke and B. Damaschke, Rev. Sci. Instrum. 67, 2957 (1996).

${ }^{15}$ T. D. Stowe, K. Yasumura, T. W. Kenny, D. Botkin, K. Wago, and D. Rugar, Appl. Phys. Lett. 71, 288 (1997).

${ }^{16}$ B. Michel, W. Mizutani, R. Schierle, A. Jarosch, W. Knop, H. Benedickter, W. Bächtold, and H. Rohrer, Rev. Sci. Instrum. 63, 4080 (1992).

${ }^{17}$ E. A. Ash and G. Nicholls, Nature 237, 510 (1972).

${ }^{18}$ A. Imtiaz, T. M. Wallis, and P. Kabos, IEEE Microwave Mag. 15, 52 (2014).

${ }^{19}$ S. M. Anlage, D. E. Steinhauer, B. J. Feenstra, C. P. Vlahacos, and F. C. Wellstood, Microwave Superconductivity (Springer, Dordrecht, 2001), pp. $239-269$.

${ }^{20}$ L. Chusseau, P. Payet, and J. Raoult, Proceedings of the 41 st International Conference on Infrared, Millimeter, and Terahertz Waves, Copenhagen, Denmark, 25-30 September (2016), pp. 1-2.

${ }^{21}$ T. L. Cocker et al., Nat. Photonics 7, 620 (2013).

${ }^{22}$ S. V. Kalinin and A. Gruverman, Scanning Probe Microscopy: Electrical and Electromechanical Phenomena at the Nanoscale (Springer, New York, 2007).

${ }^{23}$ H. Happy, K. Haddadi, D. Theron, T. Lasri, and G. Dambrine, IEEE Microwave Mag. 15, 30 (2014).

${ }^{24}$ T. Dargent, K. Haddadi, T. Lasri, N. Clément, D. Ducatteau, B. Legrand, H. Tanbakuchi, and D. Theron, Rev. Sci. Instrum. 84, 123705 (2013).

${ }^{25}$ A. Tselev, N. V. Lavrik, I. Vlassiouk, D. P. Briggs, M. Rutgers, R. Proksch, and S. V. Kalinin, Nanotechnology 23, 385706 (2012).

${ }^{26}$ S. Fabiani, D. Mencarelli, A. Di Donato, T. Monti, G. Venanzoni, A. Morini, T. Rozzi, and M. Farina, Proceedings of the IEEE/MTT-S International Microwave Symposium, Baltimore, MD, 5-10 June (2010), pp. 1-4.

${ }^{27}$ G. Anderson, Microsc. Today 21, 32 (2013).

${ }^{28}$ J. Kim, K. Lee, B. Friedman, and D. Cha, Appl. Phys. Lett. 83, 1032 (2003).

${ }^{29}$ D. E. Steinhauer, C. P. Vlahacos, S. K. Dutta, F. C. Wellstood, and S. M. Anlage, Appl. Phys. Lett. 71, 1736 (1997).

${ }^{30}$ A. Imtiaz, S. M. Anlage, J. D. Barry, and J. Melngailis, Appl. Phys. Lett. 90, 143106 (2007).

${ }^{31}$ A. Imtiaz and S. M. Anlage, Ultramicroscopy 94, 209 (2003).

${ }^{32}$ A. Tselev, N. V. Lavrik, A. Kolmakov, and S. V. Kalinin, Adv. Funct. Mater. 23, 2635 (2013).

${ }^{33}$ K. Lai, W. Kundhikanjana, M. Kelly, and Z. X. Shen, Rev. Sci. Instrum. 79, 63703 (2008).

${ }^{34}$ Y. Xing, J. Myers, O. Obi, N. X. Sun, and Y. Zhuang, J. Electron. Mater. 41, 530 (2012).

${ }^{35}$ S. Berweger et al., Nano Lett. 15, 1122 (2015).

${ }^{36}$ S. Wu and J.-J. Yu, Appl. Phys. Lett. 97, 202902 (2010).

${ }^{37}$ C. H. Joseph, G. M. Sardi, S. S. Tuca, G. Gramse, A. Lucibello, E. Proietti, F. Kienberger, and R. Marcelli, J. Magn. Magn. Mater. 420, 62 (2016).

${ }^{38}$ K. Lai, W. Kundhikanjana, H. Peng, Y. Cui, M. A. Kelly, and Z. X. Shen, Rev. Sci. Instrum. 80, 43707 (2009).

${ }^{39}$ S. Berweger, P. T. Blanchard, M. D. Brubaker, K. J. Coakley, N. A. Sanford, T. M. Wallis, K. A. Bertness, and P. Kabos, Appl. Phys. Lett. 108, 73101 (2016).

${ }^{40}$ Y. J. Oh, H.-P. Huber, M. Hochleitner, M. Duman, B. Bozna, M. Kastner, F. Kienberger, and P. Hinterdorfer, Ultramicroscopy 111, 1625 (2011).

${ }^{41}$ J. Park, S. Hyun, A. Kim, T. Kim, and K. Char, Ultramicroscopy 102, 101 (2005).

${ }^{42}$ M. Farina, A. Di Donato, T. Monti, T. Pietrangelo, T. Da Ros, A. Turco, G. Venanzoni, and A. Morini, Appl. Phys. Lett. 101, 203101 (2012). 
${ }^{43}$ M. C. Biagi, R. Fabregas, G. Gramse, M. van der Hofstadt, A. Juarez, F. Kienberger, L. Fumagalli, and G. Gomila, ACS Nano 10, 280 (2016).

${ }^{44}$ A. Tselev, J. Velmurugan, A. V. Ievlev, S. V. Kalinin, and A. Kolmakov, ACS Nano 10, 3562 (2016).

${ }^{45}$ C. Plassard, E. Bourillot, J. Rossignol, Y. Lacroute, E. Lepleux, L. Pacheco, and E. Lesniewska, Phys. Rev. B 83, 121409(R) (2011).

${ }^{46}$ J. Smoliner, H. P. Huber, M. Hochleitner, M. Moertelmaier, and F. Kienberger, J. Appl. Phys. 108, 64315 (2010).

${ }^{47}$ G. Gramse et al., Sci. Adv. 3, e1602586 (2017).

${ }^{48}$ A. Tselev, I. N. Ivanov, N. V. Lavrik, A. Belianinov, S. Jesse, J. P. Mathews, G. D. Mitchell, and S. V. Kalinin, Fuel 126, 32 (2014).

${ }^{49}$ G. Gramse, M. Kasper, L. Fumagalli, G. Gomila, P. Hinterdorfer, and F. Kienberger, Nanotechnology 25, 145703 (2014).

${ }^{50}$ V. V. Talanov and A. R. Schwartz, IEEE Trans. Microwave Theory 57, 1224 (2009).

${ }^{51}$ K. Torigoe, M. Arita, and T. Motooka, J. Appl. Phys. 112, 104325 (2012).

${ }^{52}$ A. Imtiaz et al., J. Appl. Phys. 111, 93727 (2012).

${ }^{53}$ E. Brinciotti et al., IEEE Trans. Nanotechnol. 1, 75 (2016).

${ }^{54}$ C. Gao and X.-D. Xiang, Rev. Sci. Instrum. 69, 3846 (1998).

${ }^{55}$ A. Karbassi, D. Ruf, A. D. Bettermann, C. A. Paulson, D. W. van der Weide, H. Tanbakuchi, and R. Stancliff, Rev. Sci. Instrum. 79, 94706 (2008).

${ }^{56}$ A. P. Gregory, J. F. Blackburn, K. Lees, R. N. Clarke, T. E. Hodgetts, S. M. Hanham, and N. Klein, Ultramicroscopy 161, 137 (2016).

${ }^{57}$ R. A. Kleismit, M. K. Kazimierczuk, and G. Kozlowski, IEEE Trans. Microwave Theory 54, 639 (2006).

${ }^{58}$ T. Tai, B. G. Ghamsari, and S. M. Anlage, IEEE Trans. Appl. Supercond. 23, 7100104 (2013).

${ }^{59}$ T. Monti, A. Tselev, O. Udoudo, I. N. Ivanov, C. Dodds, and S. W. Kingman, Int. J. Miner. Process. 151, 8 (2016).

${ }^{60}$ S. Rozhok, P. Sun, R. Piner, M. Lieberman, and C. A. Mirkin, J. Phys. Chem. B 108, 7814 (2004).
${ }^{61}$ K.-H. Chung, Y.-H. Lee, and D.-E. Kim, Ultramicroscopy 102, 161 (2005).

${ }^{62}$ J. Caplan, M. Niethammer, R. M. Taylor, and K. J. Czymmek, Curr. Opin. Struct. Biol. 21, 686 (2011).

${ }^{63}$ C. Diederichs, M. Bartenwerfer, M. Mikczinski, S. Zimmermann, T. Tiemerding, C. Geldmann, H. Nguyen, C. Dahmen, and S. Fatikow, Proceedings of the Australasian Conference on Robotics and Automation (ACRA), Sydney, Australia, December (2013), p. 8.

${ }^{64}$ M. F. Wieghaus, T. Tiemerding, O. C. Haenssler, and S. Fatikow, Proceedings of the International Conference on Manipulation, Automation and Robotics at Small Scales, Paris, France, 18-22 July (2016), pp. 1-6.

${ }^{65}$ A. E. H. Love, Proc. London Math. Soc. s2-22, 337 (1924).

${ }^{66}$ M. I. Elmasry, IEEE Electron Devices Lett. 3, 6 (1982).

${ }^{67}$ C. P. Yuan and T. N. Trick, IEEE Electron Devices Lett. 3, 391 (1982).

${ }^{68}$ T. Sakurai and K. Tamaru, IEEE Trans. Electron Devices 30, 183 (1983).

${ }^{69}$ N. P. van der Meijs and J. T. Fokkema, Integration 2, 85 (1984).

${ }^{70}$ A. E. Ruehli and P. A. Brennan, IEEE J. Solid-State Circuits 10, 530 (1975).

${ }^{71}$ R. C. Batra, M. Porfiri, and D. Spinello, J. Microelectromech. Syst. 15, 1175 (2006).

${ }^{72}$ E. Barke, IEEE Trans. Comput. Aided Des. 7, 295 (1988).

${ }^{73}$ W.-C. Chuang, C.-W. Wang, W.-C. Chu, P.-Z. Chang, and Y.-C. Hu, J. Micromech. Microeng. 22, 25015 (2012).

${ }^{74}$ W. H. Chang, IEEE Trans. Microwave Theory 24, 608 (1976).

${ }^{75}$ O. C. Haenssler, A. Kostopoulos, G. Doundoulakis, E. Aperathitis, S. Fatikow, and G. Kiriakidis, Proceedings of the International Conference on Manipulation, Automation and Robotics at Small Scales, Montreal, QC, Canada, 17-21 July (2017), pp. 1-5.

${ }^{76}$ M. Farina, D. Mencarelli, A. Di Donato, G. Venanzoni, and A. Morini, IEEE Trans. Microwave Theory 59, 2769 (2011).

${ }^{77}$ L. Michalas, F. Wang, C. Brillard, N. Chevalier, J. M. Hartmann, R. Marcelli, and D. Theron, Appl. Phys. Lett. 107, 223102 (2015). 